DOI: 10.34185/1991-7848.itmm.2020.01.021

\title{
О ПОСТАНОВКАХ ОБРАТНЫХ ЗАДАЧ
}

Меньшиков Ю.Л., к.т.н, доцент

Днепровский национальный университет им.Олеся Гончара, Украина

Ключевые слова: ОБРАТНЫЕ ЗАДАЧИ, ПОСТАНОВКА, СИНТЕЗ, РАСПОЗНАВАНИЕ.

Большое разнообразие обратных задач и методов их решения диктует необходимость построения некоторой систематизации обратных задач с целью рационального выбора метода решения и с целью правильной интерпретации результатов.

Будем рассматривать обратную задачу, как задачу решения операторного уравнения

$$
A z=B x=u,
$$

где $z \in Z, u \in U, x \in X$ есть некоторые метрические функциональные пространства; $A, B$ есть операторы, $A:(Z \rightarrow U), B:(X \rightarrow U)$. Предполагается, что оператор $A$ является компактным, $\quad$ а оператор $B$ непрерывный $\quad$ и ограниченный.

При решении обратных задач вместо точных исходных данных $A_{e x}, B_{e x}, x_{e x}$ задаются лишь некоторые их приближения $\tilde{A}, \tilde{B}, \tilde{x}$ :

$$
\begin{gathered}
\rho_{X}\left(\tilde{x}, x_{e x}\right) \leq \delta_{0}, \\
\rho_{A}\left(\tilde{A}, A_{e x}\right) \leq \frac{\sup _{z \in Z} \rho_{U}\left(\tilde{A} z, A_{e x} z\right)}{\rho_{Z}(z, 0)}=h, \rho_{Z}(z, 0) \neq 0, \rho_{Z}(z, 0) \leq M-\text { const }, \\
\rho_{B}\left(\tilde{B}, B_{e x}\right) \leq \frac{\sup _{x \in X} \rho_{U}\left(\tilde{B} x, B_{e x} x\right)}{\rho_{X}(z, 0)}=d, \rho_{X}(x, 0) \neq 0, \rho_{X}(x, 0) \leq \delta_{0} .
\end{gathered}
$$

где $x_{e x}$ есть точная функция в уравнении (1), $A_{e x}, B_{e x}$ есть точные операторы в (1). Функция $x_{e x}$, как правило, определяется из эксперимента с некоторой погрешностью $\delta_{0}$. Величины $h$ и $d$ определяются свойствами точных операторов $A_{e x}, B_{e x}$.

Анализ различных обратных задач показывает, что при одних и тех же исходных данных (операторы $\tilde{A}, \tilde{B}$ и функция $\tilde{x}$, конечные цели решения 
Ministry of Education and Science of Ukraine

The National Metallurgical Academy of Ukraine, Dnipro, 17 - 19 March, 2020

уравнения (1) могут быть принципиально различные. Рассмотрим некоторые возможные постановки обратных задач.

\section{1. Синтез адекватного математического описания.}

Конечной целью решения многих обратных задач является построение адекватного математического описания физического процесса с использованием данных эксперимента [1]. Далее, для простоты изложения будем полагать, что оператор $\tilde{B}$ задан точно, т.е. $\tilde{B}=B_{e x}=B$.

Конечной целью решения обратных задач такого типа является синтез математического описания для обоснованного использования результатов математического моделирования при построении прогнозов поведения физического процесса, а также для управления этим процессом [2].

В процессе решения обратных задач такого типа не возникает необходимость в учете погрешности приближенного оператора $\tilde{A}$. Обозначим совокупность всех возможных операторов как $\{\tilde{A}\}=K_{A}$. Для целей математического моделирования можно не учитывать погрешность оператора. При этом оператор $A_{a d}$ может значительно отличаться от точного оператора $A_{e x}$, а функция $z_{a d}$ может не иметь ничего общего с точным решением $z_{\text {ex }}$ уравнения (1). В силу этого отпадает потребность требовать от приближенного решения уравнения (1) сходимости к точному решению уравнения $\tilde{A} \tilde{z}_{e x}=B x_{e x}=u_{e x}$, так как функция $\tilde{z}_{e x}$ лишена физического смысла.

Если учитывать погрешность оператора $\tilde{A}$, тогда при фиксированных величинах $h, \delta_{0}$ кардинально ухудшается качество математического моделирования и возникнут проблемы с интерпретацией полученного по выбранному алгоритму приближенного решения. При этом алгоритм решения обратной задачи такого типа должен обладать свойством устойчивости к малым изменениям исходных данных [3].

\section{2. Обратные задачи измерения.}

Конечной целью решения обратных задач такого типа является получение полезной информации о реальных свойствах функции $z_{\text {ex }}$ физического процесса [4,5]. Такие задачи называют еще обратными задачами распознавания или обратными задачами интерпретации. 
International scientific and technical conference Information Technologies in Metallurgy and Machine building - ITMM 2020

Для достижения поставленной цели необходимо в обязательном порядке знать точный оператор $A_{\text {ex }}$, чтобы гарантировать принадлежность элемента $A_{e x} z_{e x}$ множеству $U_{\delta_{0}}=\left\{u: \rho_{U}\left(B^{-1} u, x_{e x}\right) \leq \delta_{0}\right\}$. Очевидно, что использование только функций из множества $U_{\delta_{0}}$ при решении обратной задачи могут дать информацию о точном решении обратной задачи. Если использовать возможные приближенные операторы $\tilde{A}$, тогда получить даже приближение к функции $z_{e x}$ невозможно с гарантией.

Если задана величина погрешности $h$ оператора $\tilde{A}$ по отношению к точному оператору $A_{e x}$, тогда возможно построить специальные регуляризирующие алгоритмы построения приближенного решения $\tilde{z}$ уравнения (1), обладающего свойством $\tilde{z} \underset{h \rightarrow 0, \delta_{0} \rightarrow 0}{\rightarrow} z_{e x}[3]$.

Однако, точный оператор $A_{e x}$ в уравнении (1) неизвестен, также как неизвестна и величина $h$.

В истории известны примеры успешного решения обратных задач измерения, в которых не использовался точный оператор $A_{e x}$ и не использовалась погрешность $h$ [4,5]. Это противоречие можно объяснить случайным попаданием элемента $\tilde{A}_{0} z_{e x}$ в множество $U_{\delta_{0}}$ с некоторым оператором $\tilde{A}_{0}$ из множества $K_{A}$. Другой вариант попадания элемента $\tilde{A}_{0} z_{e x}$ в множество $U_{\delta_{0}}$ возможен в случае, когда точное решение $z_{e x}$ известно заранее, а оператор $\tilde{A}_{0}$ выбирается подбором.

В практике решения обратных задач измерения имеются примеры приближенного решения, которые имеют важное практическое значение: медицинская диагностика (рентгеновские, томографические, УЗИ исследования), техническая диагностика и т.д. [4,5]. Однако, рассмотрение, например, рентгеновских снимков не дает основания полагать их приближенным отражением реальности, так как тогда точная картина будет абсурдной. Скорее всего, такие решения являются лишь оценками точных решений в определенном смысле [6]. 


\section{References}

1. Yu.L.Menshikov. Criteria for estimation the adequacy of mathematical descriptions of physical processes // Chapter, Modeling and Simulation in Engineering, Intech-Open, London, 2019, 19p.

2. Н.Н. Красовский, Теория управления движением, Наука, Москва, 1968, -478стр.

3. А.Н.Тихонов, В.Я.Арсенин. Методы решения некорректных задач. М.; Наука, 1972, 184 стр.

4. Е.А.Гребеников, Ю.А.Рябов. Поиски и открытия планет. М.: Наука, 1984. -224 с.

5. V. Chandola, A. Banerjee and V. Kumar, Anomaly detection: A survey, ACM Computing Surveys (CSUR), 41 (2008): 15: 1-15:58.

6. Yu.L.Menshikov. One approach to solutions of measurement's inverse problems // Journal Mathematical Inverse Problems, Vol.1, No.2, 2014, USA, p.71-85.

\section{ON THE FORMULATION OF INVERSE PROBLEMS}

\section{Menshikov Yurii}

Abstract. Some possible options for the formulation of inverse problems are considered. The ultimate research goals in these cases determine the algorithms for the approximate solution of the inverse problem and allow one to correctly interpret these solutions. Two main statements of inverse problems considered: inverse problems of synthesis and inverse problems of measurement. It is shown that in inverse synthesis problems one should not take into account the error of the mathematical model. In addition, it is possible in these cases to synthesize approximate solution algorithms that do not have a regularizing property. Examples of practical problems considered.

Keywords: INVERSE PROBLEMS, FORMULATION, SYNTHESIS, RECOGNITION. 